\title{
Schilddrüsenknoten: ATA-Leitlinie aus europäischer Sicht
}

Karl Michael Derwahl, Peter Goretzki

\author{
Die überarbeitete Leitlinie der American Thyroid Association (ATA) von 2015 [1] be- \\ zieht sich auf ein Land mit im Durchschnitt normaler Jodversorgung. Bei der Abklärung \\ von Schilddrüsenknoten muss jedoch die Jodversorgung berücksichtigt werden. In \\ einer kürzlich publizierten Stellungnahme gehen europäische und amerikanische \\ Experten auf die besonderen Verhältnisse in ehemaligen Jodmangelgebieten in Europa \\ ein [4], wozu auch Deutschland gehört [2].
}

\section{Hintergrund}

Die American Thyroid Association (ATA) hat 2015 hat eine grundlegend überarbeitete Leitlinie der vorausgehenden Fassung von 2009 publiziert [1]. Die von amerikanischen Thyreologen unter Beteiligung von 2 europäischen Kollegen verfassten Leitlinien reflektieren die Verhältnisse in einem Land mit im Durchschnitt normaler Jodversorgung. In den USA sind die meist kleineren Schilddrüsenknoten in der Regel als solitäre Knoten und selten in einer Knotenstruma nachweisbar und Autonomien sehr selten. In der Abklärung von Schilddrüsenknoten muss jedoch die Jodversorgung berücksichtigt werden. Auch wenn die Jodversorgung in Deutschland insgesamt noch nicht optimal ist, gehört unser Land nicht mehr zu den Jodmangelgebieten [2]. Als Folgen des Jodmangels weisen aber immer noch ca. 25 \% der Bevölkerung Schilddrüsenknoten oder eine Knotenstruma auf [2]. Kürzlich wurde eine Stellungnahme europäischer Nuklearmediziner, Endokrinologen und endokriner Chirurgen unter Beteiligung amerikanischer Kollegen publiziert, die auf die besonderen Verhältnisse in ehemaligen Jodmangelgebieten in Europa eingeht und die amerikanischen Leitlinien unter Berücksichtigung der europäischen Gegebenheiten kommentiert [4].

\section{Epidemiologie des malignen Schilddrüsenknotens}

Den mehr als 20 Millionen in Deutschland lebenden Menschen, die nach Hochrechnung einen (möglicherweise nie diagnostizierten) Schilddrüsenknoten aufweisen, steht eine vergleichsweise geringe Anzahl von Schilddrüsenkarzinomen gegenüber. Nach der Inzidenzstatistik des Robert-Koch-Instituts (www.krebsdaten.de) erkrankten 2016 in Deutschland 7780 Patienten (5280 Frauen und 2500 Männer) an einem Schilddrüsenkarzinom
( $\triangleright$ Tab. 1). Die 10-Jahres-Prävalenz dieses Karzinoms beträgt bei Frauen 42400 und bei Männern 16 400. Mit einer 10-Jahres-Überlebensrate von $91 \%$ bei Frauen und $86 \%$ bei Männern ist die Prognose des differenzierten Schilddrüsenkarzinoms im Vergleich zu anderen Karzinomen sehr gut.

Die geringe jährliche Inzidenz von Schilddrüsenkarzinomen bei 20 Millionen Schilddrüsenknoten (wegen des Auftretens multipler Knoten ist die Gesamtzahl der Knoten sogar noch sehr viel höher!) macht deutlich, warum bei der Abklärung des Schilddrüsenknotens die ATA-Leitlinien nicht uneingeschränkt übernommen werden können, sondern die besonderen Verhältnisse in einem ehemaligen Jodmangelgebiet berücksichtigt werden müssen.

Nach der Statistik des Robert-Koch-Instituts ist die Erkrankungsrate des differenzierten Schilddrüsenkarzinoms in den letzten Jahren besonders bei Frauen deutlich gestiegen (www.krebsdaten.de). Auf der anderen Seite ist die Sterberate leicht gefallen. Dies wird durch die Detektion von vorwiegend papillären Mikrokarzinomen (Durchmesser $<1 \mathrm{~cm}$ ) erklärt. Diese Zunahme von Mikrokarzinomen findet sich auch in Ländern mit ausreichender Jodversorgung und aufgrund der guten Prognose wird vom Nachweis solcher Mikrokarzinome im höheren Alter abgeraten [5].

In Bezug auf die Inzidenz des differenzierten Schilddrüsenkarzinoms als solitärer Knoten und in der Knotenstruma ist zu beachten: Noch in den 1980er-Jahren entwickelten sich Schilddrüsenkarzinome in bis zu $40 \%$ der Fälle in multinodösen Strumen; diese wurden meist zufällig bei der histologischen Aufarbeitung von Operationspräparaten diagnostiziert [6]. In späteren Studien war dieser Anteil zwar geringer, aber immer noch weitaus höher als in den USA [7]. 
- Tab. 1 Inzidenz des Schilddrüsenkarzinoms 2014 nach ICD-10 (Aus: Robert-Koch-Institut; www.krebsdaten.de).

\begin{tabular}{|l|l|l|}
\hline Parameter & Männer & Frauen \\
\hline Neuerkrankungen & 2500 & 5280 \\
\hline Erkrankungen/100 000 & 6,2 & 12,7 \\
\hline Sterbefälle & 286 & 390 \\
\hline 10-Jahres-Prävalenz & 16400 & 42400 \\
\hline 10-Jahres-Überlebensrate & $86 \%$ & $91 \%$ \\
\hline
\end{tabular}

- Tab. 2 Schilddrüsenkarzinome nach histologischem Typ und Geschlecht, ICD-10 2013-2014 (Robert-Koch-Institut; www.krebsdaten.de).

\begin{tabular}{|l|c|c|}
\hline Histologie & Männer & Frauen \\
\hline papilläre Karzinome & $68 \%$ & $78 \%$ \\
\hline follikuläre Karzinome & $14 \%$ & $11 \%$ \\
\hline medulläre Karzinome & $7 \%$ & $4 \%$ \\
\hline anaplastische Karzinome & $4 \%$ & $2 \%$ \\
\hline andere Morphologien & $2 \%$ & $1 \%$ \\
\hline ohne nähere Angaben & $5 \%$ & $4 \%$ \\
\hline
\end{tabular}

\section{Histologie und Bedeutung des Jodmangels}

\section{Histologie der Schilddrüsenkarzinome}

Durch die Verbesserung der Jodversorgung hat sich die Prävalenz der histologischen Entitäten des differenzierten Schilddrüsenkarzinoms verschoben. Durch die Beseitigung des Jodmangels hat der relative Anteil des follikulären Karzinoms zugunsten des prognostisch günstigeren papillären Karzinoms abgenommen [8].

Nach der ICD-Statistik verteilten sich 2015/2016 die differenzierten Schilddrüsenkarzinome bei Frauen auf $78 \%$ papilläre Karzinome, $11 \%$ follikuläre Karzinome und 4\% medulläre Karzinome und bei Männern auf $68 \%$ papilläre Karzinome, $14 \%$ follikuläre Karzinome und $7 \%$ medulläre Karzinome ( $\triangleright$ Tab. 2).

\section{Diagnostik}

\section{TSH, Autonomie und Szintigrafie}

Nach den Leitlinien der ATA (hoher Empfehlungsgrad) muss bei jedem Schilddrüsenknoten initial der TSH gemessen werden und bei erniedrigtem TSH, nicht aber bei normalem oder erhöhtem TSH, eine I-123-Szintigrafie durchgeführt werden. Einige Studien in Jodmangelgebieten haben gezeigt, dass auch bei einem niedrig normalen TSH-Wert eine Autonomie vorliegen kann [9, 10]. Auch wenn in Deutschland die Autonomie als Folge der besseren Jodversorgung seltener wird, muss dies bis heute berücksichtigt werden. In den USA ist aufgrund der Jodversorgung die Autonomie selten. Für die Szintigrafie wird in Deutschland in der Regel Technetium-99m verwandt. Gegenüber Jodid-123 hat es den Vorteil einer geringeren Halbwertszeit und einer geringeren Strahlendosis.

In Deutschland wird unabhängig vom TSH in der diagnostischen Abklärung des Schilddrüsenknotens $>1 \mathrm{~cm}$, zumindest präoperativ, eine Technetium-99m-Szintigrafie durchgeführt. Dies ist in den USA nicht der Fall.

\section{Calcitoninbestimmung beim Schilddrüsenknoten}

Dieses Thema wird in derselben Ausgabe im Beitrag „Das medulläre Schilddrüsenkarzinom und die multiple endokrine Neoplasie Typ 2“ (DOI https://doi.org/10.1055/ a-1005-8798) behandelt.

\section{Sonografie, Malignitätskriterien und Indika- tionen zur Feinnadelaspirationspunktion}

In Deutschland wird die Schilddrüsensonografie mit sehr weit gestellter Indikation durchgeführt, z. B. zum Nachweis eines Knotens oder allgemein bei Schilddrüsenvergrößerung. Im Gegensatz dazu empfehlen die ATA-Leitlinien eine Sonografie der Schilddrüse nur bei einem suspekten Schilddrüsenknoten, einer Knotenstruma oder einem als Nebenbefund entdeckten abnormen Knoten im CT bzw. MRT oder einer Speicherung in der Schilddrüse bei FDG-18-PET-Scan, das in der Regel aus anderer Indikation durchgeführt wurde [1]. Ergänzend zu dieser Sonografie muss in jedem Fall eine sonografische Evaluierung der Halslymphknoten erfolgen.

In Analogie zum Thyroid Imaging Reporting and Data System (TI-RADS; [13]), einer Standardisierung der sonografischen Untersuchung von Schilddrüsenknoten zur Evaluierung des relativen Malignitätsrisiko, hat die ATA in ihrer Leitlinie 5 Klassen definiert, diese mit typischen sonografischen Befunden belegt und jeweils das prozentuale Malignitätsrisiko angegeben: benigner Knoten ( $0 \%$ Risiko), sehr geringer Malignitätsverdacht $(<3 \%)$, geringer Verdacht (5-10\%), intermediärer Verdacht (10-20\%) und hoher Verdacht (>70-90\%) ( Tab. 3).

Der Risikobewertung liegen dabei mehrere Malignitätskriterien zugrunde:

- solider Knoten,

- ausgeprägte Echoarmut,

- irregulärer Knotenrand,

- Mikrokalzifizierung sowie

- Knoten tiefer als breit. 
- Tab. 3 Ultraschallmuster, geschätztes Malignitätsrisiko und Indikation zur Feinnadelpunktion (FNA) (nach ATA-Leitlinien 2015 [1]).

\begin{tabular}{|c|c|c|c|}
\hline Bewertung & Ultraschallmuster & Malignitätsrisiko & FNA ab Größe \\
\hline hochgradig verdächtig & $\begin{array}{l}\text { echoarmer Knoten oder echoarmer Anteil eines zystischen } \\
\text { Knotens mit } 1 \text { oder mehreren Merkmalen: } \\
\text { - irregulärer Rand (infiltrativ, gelappt) } \\
\text { - Mikrokalizifikationen, tiefer als breit } \\
\text { - Randkalzifikation mit verdrängendem Gewebestrang } \\
\text { - extrathyreoidale Ausdehnung }\end{array}$ & $>70-90 \%$ & FNA bei Knoten $\geq 1 \mathrm{~cm}$ \\
\hline intermediär verdächtig & $\begin{array}{l}\text { echoarmer Knoten mit glattem Rand ohne Mikrokalzifika- } \\
\text { tionen, extrathyreoidale Ausdehnung, tiefere als breitere } \\
\text { Ausdehnung }\end{array}$ & $10-20 \%$ & FNA bei Knoten $\geq 1 \mathrm{~cm}$ \\
\hline wenig verdächtig & $\begin{array}{l}\text { echogleicher oder echoreicher Knoten oder zystischer } \\
\text { Knoten mit exzentrischem solidem Anteil, ohne Mikrokalzi- } \\
\text { fikationen, ohne irregulären Rand, ohne extrathyreoidale } \\
\text { Ausdehnung, ohne tiefere als breitere Form }\end{array}$ & $5-10 \%$ & FNA bei Knoten $\geq 1,5 \mathrm{~cm}$ \\
\hline sehr wenig verdächtig & $\begin{array}{l}\text { spongiformer oder partiell zystischer Knoten ohne unter } \\
\text { hochgradig, intermediär oder wenig verdächtig beschrie- } \\
\text { bene Merkmale }\end{array}$ & $<3 \%$ & $\begin{array}{l}\text { FNA bei Knoten } \geq 2 \mathrm{~cm} \\
\text { erwägen oder beobachten }\end{array}$ \\
\hline gutartig & rein zystischer Knoten & $<1 \%$ & keine FNA \\
\hline
\end{tabular}

- Tab.4 Ultraschallkriterien des European Thyroid Imaging Reporting and DATA-Systems und FNA-Indikationen (aus: EU-TIRADS [14]).

\begin{tabular}{|c|c|c|c|}
\hline Kategorie & Ultraschall & Malignitätsrisiko & FNA-Indikation \\
\hline EU-TIRADS 1: normal & kein pathologischer Befund & $0 \%$ & keine \\
\hline EU-TIRADS 2: benigne & Zyste, spongiform & ca. $0 \%$ & keine \\
\hline EU-TIRADS 3: niedriges Risiko & oval, glatt, echogleich, echoreich, keine Hochrisiko-Zeichen & $2-4 \%$ & Knoten $>2 \mathrm{~cm}$ \\
\hline EU-TIRADS 4: intermediäres Risiko & oval, glatt, leicht echoarm, keine Hochrisiko-Zeichen & $6-17 \%$ & Knoten $>1,5 \mathrm{~cm}$ \\
\hline EU-TIRADS 5: hohes Risiko & $\begin{array}{l}\text { mindestens } 1 \text { Zeichen: } \\
\text { - irregulärer Rand } \\
\text { - irreguläres Bild } \\
\text { - Mikrokalzifikationen } \\
\text { - ausgeprägt echoarm }\end{array}$ & $26-87 \%$ & $\begin{array}{l}\text { Knoten }>1,0 \mathrm{~cm} \text {, } \\
\text { ggf. }<1,0 \mathrm{~cm}\end{array}$ \\
\hline
\end{tabular}

\section{Merke}

Die große Lücke zwischen den von der ATA angegebenen Risiken bei intermediärem und bei hohem Verdacht macht deutlich, welche große Bedeutung der Erfahrung des Untersuchers zukommt!

Eine Feinnadelaspirationspunktion (FNA-Punktion) wird von der ATA empfohlen [1]

- bei intermediärem Verdacht und hohem Verdacht bei allen Knoten > 1 cm Durchmesser,

- bei geringem Verdacht bei einem Durchmesser $>1,5 \mathrm{~cm}$ und

- bei sehr geringem Verdacht bei einem Durchmesser $>2 \mathrm{~cm}$.
Bei diesen Empfehlungen muss betont werden, dass sie für solitäre Knoten in einem Land mit normaler Jodversorgung gelten und nicht für Knotenstrumen. Zudem gilt, dass in ehemaligen Jodmangelgebieten die Knotendurchmesser größer sind, sodass in Deutschland bei vielen Knoten mit geringem oder sogar sehr geringem Verdacht eine FNA-Punktion durchgeführt werden müsste, was sicherlich nicht indiziert ist.

Europäische Thyreologen haben analog dem amerikanischen System das EU-TIRADS-System mit den Kategorien 1 (normal), 2 (benigne), 3 (niedriges Risiko), 4 (intermediäres Risiko) und 5 (hohes Risiko) entwickelt, das etwas einfacher ist ( Tab.4; [14]). In der Kategorie 5 wird bei EU-TIRADS ein Risiko von 26-87\% angegeben. 
Analog zur ATA hat die ETA zur Kategorisierung im EUTIRADS-System für die Indikation zur FNA eine Risikostratifizierung erstellt ( $\triangleright$ Tab. 4). Diese berücksichtigt in ihrem Algorithmus, wie die ATA, die Malignitätskriterien und Echogenität von Knoten, wobei allerdings zwischen leicht echoarm und ausgeprägt echoarm unterschieden wird. Da auch die Mehrzahl der benignen Knoten in ehemaligen Jodmangelgebieten echoarm ist, ist die ausgeprägte Echoarmut ein wesentliches Merkmal für Malignität [15].

\section{Abklärung der Knoten in einer Knotenstruma}

In ehemaligen Jodmangelgebieten überwiegen bei den üngeren die solitären Knoten gegenüber Knoten in einer Struma bzw. einer Knotenstruma. In einer großen Studie fand sich bei der Auswertung von 32120 FNA-Zytologien nur bei den jüngeren Patienten ein papilläres Karzinom als solitärer Knoten, während jenseits des 50. Lebensjahres ein Karzinom gleich häufig oder sogar etwas häufiger in einer Struma nachweisbar war [16]. Häufig werden allerdings Karzinome in einer Struma erst postoperativ bei der histologischen Aufarbeitung des Resektats diagnostiziert [7].

Gemäß der ATA-Leitlinien soll die Abklärung des Knotens in einer Struma nodosa analog dem Prozedere beim solitären Knoten erfolgen [1]. Es gelten dieselben sonografischen Kriterien und dieselben Volumenangaben zur FNAPunktion wie bei solitären Knoten. In Gebieten mit Jodmangel und in Deutschland finden sich aber häufig bei älteren Patienten neben solitären Knoten zu sogenannten Konglomerattumoren miteinander verwachsene Knoten. In solchen Knoten finden sich regelmäßig Verkalkungen. Bei ineinander gewachsenen Knoten sind häufig auch die Ränder unscharf oder nicht mehr nachweisbar.

Auch das Kriterium eines Knotenvolumens $\geq 2 \mathrm{~cm}$ als Indikation für eine FNA-Punktion ist für diese multinodösen Strumen nicht anwendbar.

\section{Abklärung eines Knotens in der Schwangerschaft}

Da es keine Populationsstudie über eine höhere Malignität von in der Schwangerschaft entdeckten Knoten gibt, empfiehlt die ATA dieselbe diagnostische Abklärung wie bei anderen Knoten [1]. In jedem Fall sollten die in der Schwangerschaft meist nur leicht wachsenden Knoten sonografisch überwacht werden. Wenn eine nach Leitlinien indizierte FNA-Punktion zytologisch ein differenziertes Schilddrüsenkarzinom ergibt, sollte nur bei substanziellem Wachstum des Tumors und/oder bei Lymphknotenbefall im Einzelfall eine Operation (s. unten) erfolgen.

Wenn bei langsamem Wachstum oder nichtsignifikanter Zytologie (s. unten) eine Thyreoidektomie auf die Zeit nach der Geburt verschoben wird, sollte nach ATA-Leitlinien eine Schilddrüsenhormontherapie mit einem TSHZielwert von 0,3-2,0 mU/l für die gesamte verbleibende Schwangerschaft erfolgen (geringer Empfehlungsgrad mit niedriger Evidenz). Begründet wird diese Empfehlung mit einer möglichst geringen Stimulation der Schilddrüse und damit des Tumors bei niedrigerem TSH-Wert. Diese Empfehlung basiert auf einer Studie [17], die eine positive Korrelation zwischen einem fortgeschrittenen Tumorstadium des differenzierten Schilddrüsenkarzinoms und höheren TSH-Spiegel nachgewiesen hat.

\section{Elastografie, Farbdoppler-Sonografie und MIBI-Szintigrafie}

Die Elastografie zur Charakterisierung der Steifheit von Knoten als Hinweis für Malignität wird in den ATA-Leitlinien 2015 nicht allgemein empfohlen. Als wesentliche Gründe werden die geringe Verbreitung von Ultraschallgeräten mit einem Elastografie-Modul und divergierende Ergebnisse in seinerzeit publizierten Studien angegeben [1]. Allerdings wird von der ATA eingeräumt, dass im Einzelfall bei der exakten Evaluierung eines Knotens die Elastografie durchaus hilfreich sein kann. Die ETA-Leitlinie zur Sonografie des Schilddrüsenknotens stimmt mit der ATA überein, dass die Elastografie die klassische Sonografie nicht ersetzen kann [14]. Die Autoren der Leitlinien sehen die Elastografie als komplementäres Verfahren und betonen den negativen prädiktiven Wert zum Ausschluss von Malignität bei sehr weichen Knoten.

Die Farbdoppler-Sonografie zur Untersuchung der Knotenperfusion wird in den ATA-Leitlinien zur Evaluierung des Schilddrüsenknotens nicht direkt empfohlen. Die vermehrte Vaskularisierung eines Knotens wird zwar als Risikofaktor bezeichnet, der aber nicht „robust“ ist. In der Beurteilung von Lymphknoten wird ferner die periphere Vaskularisierung als Malignitätskriterium erwähnt [18]. Die Sonografie-Leitlinie der ETA betont zwar, dass maligne Knoten eine ausgeprägtere intranodale Vaskularisierung im Vergleich zu benignen Knoten aufweisen, empfiehlt aber die Farbdoppler-Untersuchung für die Evaluierung des Schilddrüsenknotens allenfalls zur Abgrenzung eines isoechogenen Knotens [14]. Gegen den Routineeinsatz der Farbdoppler-Technik spricht ferner die unterschiedliche Sensitivität in Abhängigkeit vom UItraschallgerät und die Abhängigkeit des Perfusionssignals von der individuellen Einstellung der Intensität [4].

Die MIBI-Szintigrafie, die gelegentlich zur Evaluierung des Schilddrüsenknotens in Deutschland zur Anwendung kommt, hat zwar einen hohen negativen prädiktiven Wert, die Spezifität ist im ehemaligen Jodmangelgebiet allerdings gering [15]. Die ATA empfiehlt sie in ihren Leitlinien nicht. 


\section{Sonografie der Lymphknotenkompartimente am Hals}

Zu jeder Schilddrüsensonografie gehört immer eine Untersuchung der Halslymphknoten zum Ausschluss bzw. Nachweis von Metastasen. Die ATA-Leitlinie bezieht sich bei der Evaluierung der Lymphknoten am Hals auf die Kriterien der ETA-Leitlinie 2013 zur Halssonografie [18]. Die europäische Kritik fügt als weitere Kriterien den Verlust des Hilus und den unregelmäßigen Rand hinzu [4]. Ferner wird auf die Notwendigkeit eines hochauflösenden Ultraschallgeräts, eines Schallkopfes $\geq 10 \mathrm{MHz}$ und eines erfahrenen Untersuchers hingewiesen. Die Ultraschallbefunde des zentralen und der lateralen Halskompartimente sollten in einem Diagramm detailliert festgehalten werden.

Als Malignitätskriterien für den malignen Lymphknotenbefall gelten zusammenfassend:

- eine runde Form (statt der typischen ovalen),

- Mikrokalzifikationen,

- zystische Aspekte,

- eine Hyperechogenität,

- eine periphere Vaskularisierung,

- ein Verlust des Hilus und

- ein irregulärer Rand.

Verdächtige Lymphknoten werden unter Ultraschallsicht mit der Feinnadel punktiert und die Aspirationsflüssigkeit wird auf Thyreoglobulin untersucht [1]. Auch suspekte Lymphknoten mit einem Durchmesser $<1 \mathrm{~cm}$ sollten nach Möglichkeit punktiert werden.

Gemäß den ATA-Leitlinien wird die durch Feinnadelaspiration (FNA) gewonnene Zytologie nach dem BethesdaSystem in 6 Kategorien unterteilt [19]:

- nichtdiagnostisches oder ungenügendes Material (Malignitätsrisiko 1-4\%),

- benigne (Malignitätsrisiko 0-3\%),

- Atypie oder follikuläre Läsion ungeklärter Signifikanz (Malignitätsrisiko 5-15\%),

- follikuläre Neoplasie oder Verdacht auf follikuläre Neoplasie (Malignitätsrisiko 15-30\%),

- Malignitätsverdacht (Malignitätsrisiko 60-75\%) sowie

- maligne (Malignitätsrisiko 97-99\%).

Das in Klammern angegebene Malignitätsrisiko kann im Einzelfall bei durchgeführter Operation in der Histologie besonders in den ersten 3 Kategorien höher sein [1].

Bei nichtsignifikanter Zytologie wird eine erneute FNAPunktion empfohlen (hoher Empfehlungsgrad mit mäßiger Evidenz). Bei wiederholter nichtsignifikanter Zytologie sollte bei geringem sonografischem Malignitätsverdacht eine engmaschige sonografische Kontrolle erfolgen oder gleich eine Operation angestrebt werden.
Bei hohem Malignitätsverdacht, einem Knotenwachstum $>20 \%$ im Verlauf oder klinischen Risikofaktoren (nicht verschieblicher Knoten, Schmerz, Heiserkeit, Husten, Lymphknotenvergrößerung oder Bestrahlung in der Vorgeschichte) wird eine Operation empfohlen [1].

Selbstverständlich sind bei der Frage der Operationsindikation (s. unten) auch bei maligner Zytologie das Gesamtrisiko des Tumors, z. B. papilläres Mikrokarzinom ohne lokales invasives Wachstum und/oder Metastasierung, und die Komorbidität zu berücksichtigen [1].

\section{Stellenwert der molekularen Diagnostik bei nichtsignifikanter Zytologie}

Die ATA-Leitlinie ist bei der Bewertung der molekularen Diagnostik zurückhaltend, bewertet sie grundsätzlich positiv, aber verweist auch auf den (noch) begrenzten Wert der verfügbaren diagnostischen Methoden in der Bewertung der nichtsignifikanten Zytologie. Erwähnt werden für das papilläre Karzinom die Mutationen und Rearrangements (BRAF, RAS, RET/PTC und PAX(/PPARgamma) und die mRNA-Expressionsprofilmuster-Analyse von wachstumsassoziierten Genen [1]. Nur in der Risikobewertung des papillären Karzinoms kann die molekulare Diagnostik einen Beitrag zur Bewertung des Malignitätsrisiko leisten, sodass bei Verfügbarkeit eine schwache Empfehlung ausgesprochen wird. Letztlich sind aber die sonografischen und klinischen Kriterien für die Entscheidung zu einer Operation maßgeblich.

Die europäischen Kommentare zu den ATA-Leitlinien bestätigen im Wesentlichen die Empfehlungen zur molekularen Diagnostik nichtsignifikanter Zytologien [4]. Sie weisen darauf hin, dass sich durch die Differenzierung einer nichtinvasiven follikulären Schilddrüsenneoplasie mit papillären nukleären Veränderungen in der neuen WHO-Klassifikation und dem Nachweis von RAS-Mutationen oder PAX8-PPRgamma-Rearrangements in benignen Schilddrüsenknoten die Bedeutung der molekularen Diagnostik weiter relativiert hat [20,21]. Allenfalls könnte bei malignitätsverdächtiger Zytologie ohne Nachweis follikulärer Muster die molekulare Diagnostik das operative Prozedere möglichweise verändern [4].

\section{Operative Strategie und weiteres Prozedere}

Die geringe Inzidenz von Malignomen in diagnostizierten Schilddrüsenknoten verlangt eine kritische Betrachtung der Operationsindikation aufgrund fraglicher Malignität.

Untersuchungen in den USA sowie in Frankreich zeigen keine Korrelation zwischen der Frequenz von Schilddrüsenoperationen und dem Auffinden von malignen Schild- 
> Tab. 5 Prä-, intra- und postoperative Diagnostik bei Verdacht auf Schilddrüsenkarzinom.

\begin{tabular}{|c|c|c|}
\hline präoperative Untersuchungen & intraoperative Untersuchung & postoperative Diagnostik \\
\hline $\begin{array}{l}\text { Serum-Calcitonin } \\
\text { Ultraschallknoten (TIRADS-Einteilung) } \\
\text { US: Halslymphknoten }\end{array}$ & $\begin{array}{l}\text { Gefrierschnitt des Knotens (Histologie; } \\
\text { Größe, R0/1, Kapsel, Gefäßinvasion, } \\
\text { multiple PTMC }\end{array}$ & $\begin{array}{l}\text { definitive Histologie } \\
\text { PTNM; mPTMC? } \\
\text { PTC Subtypen; miFTC/wiFTC; PDTC }\end{array}$ \\
\hline $\begin{array}{l}\text { Elastografie } \\
\text { Durchblutungsmessung }\end{array}$ & & Ki-67 \\
\hline $\begin{array}{l}\text { Knoten: FNA-Zytologie; plus molekulare } \\
\text { Marker (BRAF) }\end{array}$ & & $\begin{array}{l}\text { molekulare Marker (Panel: RET, BRAF, } \\
\text { TERT etc.) }\end{array}$ \\
\hline LK: FNA-Zytologie plus TG im Aspirat & Gefrierschnitt: LK & $\begin{array}{l}\text { LN 1a vs. } 1 \mathrm{~b} \\
\text { M0 vs. M1 } \\
\text { postoperatives TG/CT }\end{array}$ \\
\hline
\end{tabular}

US = Ultraschalluntersuchung; LK= Lymphknoten; FNA = Feinnadelaspiration; TG = Thyreoglobulin; PTMC = papilläry thyroid micro carcinoma; FTC = follicular thyroid cancer; PDTC = poorly differentiated thyroid carcinoma.

drüsentumoren. Die Autoren schlussfolgern, dass zumindest in diesen Ländern rein subjektive Einschätzungen die Diagnose eines „verdächtigen Knotens“ ausmachen und zur Indikation einer Operationsnotwendigkeit veranlassten. Exakte Daten für Deutschland liegen uns hierzu nicht vor und können nur auf der Basis von Daten der Versicherer subsumiert werden. Sie zeigen insgesamt, dass die Indikation zu einer „diagnostischen Schilddrüsenoperation“ eines als „verdächtig“ erachteten Schilddrüsenknotens in Deutschland sehr häufig gestellt wird, ohne dass alle Möglichkeiten präoperativer Selektion ausgenutzt werden $[23,24]$.

Umso wichtiger erscheint die Verwendung der vielfältigen Möglichkeiten einer präoperativen Einschätzung eines Schilddrüsenknotens bezüglich seiner Dignität ( $\triangleright$ Tab.5). So kann eine exakte Befundung von Schilddrüsenknoten auch schon präoperativ auf die vorzunehmende Ausdehnung der Operation hinweisen.

Die operative Strategie wird durch die zugrunde liegende Histologie (PTC: papillary thyroid cancer/FTC: follicular thyroid cancer/MTC: medullary thyroid cancer), das Alter und Geschlecht der Patienten ( $>/<55$. Lebensjahr) sowie den Ultraschallbefund des Tumors und der angrenzenden Lymphknoten (klinisch eingeschätztes TNM-Stadium (T1-3N0-1bM0-1)) bestimmt.

Ergebnisse prospektiver Langzeituntersuchungen von Registerdaten (SEER) der USA haben die ATA-Leitlinien 2015 bestimmt und weisen entscheidende Unterschiede im empfohlenen operativen Vorgehen im Vergleich zu den Leitlinien des Jahres 2009 und zu den deutschen Leitlinien auf ( $\triangleright$ Abb. 1).

Als minimales primäres Vorgehen kann die Hemithyreoidektomie akzeptiert werden, die bei klinisch nachweis- baren Lymphknotenmetastasen auf der tumortragenden Seite mit einer zentralen Lymphknotendissektion dieser Seite kombiniert wird.

Dieses Vorgehen trifft nicht für ausgedehnte Tumorgeschehen mit bewiesener Malignität zu. Bei diesen Patienten wird primär eine 1-zeitige, vollständige Entfernung des Tumors und regionaler Metastasen angestrebt.

Ausnahmen davon bilden Patienten mit nicht kurativ resektablen Tumoren und/oder ausgedehnten, nicht radikal entfernbaren Fernmetastasen sowie Patienten mit anaplastischem Schilddrüsenkarzinom. Bei diesen Patienten ist vor jeder operativen Intervention die Indikation zur Chirurgie zu überdenken und gegenüber der einer primären multimodalen Radiochemotherapie abzuwägen.

Mit vollständiger Hemithyreoidektomie kann intraoperativ anhand des Ergebnisses eines Gefrierschnitts entschieden werden, ob eine Erweiterung der Operation zu einer Thyreoidektomie oder Thyreoidektomie mit zentraler Lymphknotendissektion nötig ist. Ergibt die intraoperative histologische Untersuchung jedoch einen abgekapselten, hoch differenzierten Tumor (klassisches PTC, follikuläre Variante eines PTC, minimalinvasives FTC ohne nachgewiesene Gefäßinvasion) bis zu einem Gesamtdurchmesser von $1 \mathrm{~cm}$ (CAEK-Leitlinien) versus $4 \mathrm{~cm}$ [1] ohne makroskopisches extrathyreoidales Wachstum, ist sowohl die Hemithyreoidektomie als auch die Thyreoidektomie vertretbar. Die neue Größengrenze von $4 \mathrm{~cm}$ in den ATA-Leitlinie basiert auf SEER-Daten, die für diese Patientengruppe (PTC1b-2) keinen Unterschied im Gesamtüberleben bei unterschiedlichem operativem Vorgehen nachweisen konnte.

Die alleinige Hemithyreoidektomie schließt eine vollständige pathologische TNM-Evaluation wie auch eine nach- 


\begin{tabular}{|c|c|c|c|c|}
\hline Vorgehen & ATA Leitlinien 2015 & Ausnahmen & CAEK Leitlinien & $\begin{array}{l}\text { Begründung der ATA } \\
\text { Leitlinien }\end{array}$ \\
\hline Hemithyreoidektomie & $\begin{array}{l}\text { PTC (T1aN0-1) } \\
\text { PTC (T1b-2N0) } \\
\text { ggf. TX } \\
\text { TX }\end{array}$ & $\begin{array}{l}\text { PTC + mPTMC } \\
\text { BRAF, RET, TERT } \\
\text { Fam. PTC/FTC; } \\
\text { Ext. Bestrahlung } \\
\text { Makroskop. ETE } \\
\text { agress. Variante }\end{array}$ & Thyreoidektomie & $\begin{array}{l}\text { ÜL. Rezidive } \\
\text { Überleben } \\
\text { Persistenz } \\
\text { Rezidive } \\
\\
\text { Persistenz } \\
\text { Metastasen } \\
\text { ÜL-Rate }\end{array}$ \\
\hline Hemithyreoidektomie & $\begin{array}{l}\text { FTC (miT1a-b)N0 } \\
\text { FTC (miT2)N0 } \\
\text { TX }\end{array}$ & T1b-2 Fam FTC & $\begin{array}{l}\text { FTC (miT1a-b)N0 } \\
\text { Thyreoidektomie }\end{array}$ & ÜL. Rezidive \\
\hline Lymphknoten Diss. & PTC (Alle T; cLK+) & & PTC (alle T; cLK+) & Persistenz \\
\hline Prohylaktisch LK-D zentral & ggf. PTC (T3-4)NO & & ggf. PTC (T3-4) & $\begin{array}{l}\text { Persistenz } \\
\text { Rezidiv }\end{array}$ \\
\hline $\begin{array}{l}\text { Diagnostisch (lat. + zent.) } \\
\text { LK-FNA (Zyto + TG) + ggf. } \\
\text { Dissektion }\end{array}$ & $\begin{array}{l}\text { PTC (alle TN0) } \\
\text { mit Risikofaktoren } \\
\text { (Histo, Alter, etc.) }\end{array}$ & & keine Angabe & Rezidiv \\
\hline
\end{tabular}

Abb. 1 Unterschiede zwischen den CAEK-Leitlinien und den ATA-Leitlinien 2015. PTC= papillary thyroid cancer; FTC= follicular thyroid cancer; MTC = medullary thyroid cancer; $\mathrm{mPTMC}=$ multiple papillary micro carcinoma; LK = Lymphknoten; TG = Thyreoglobulin.

folgende Radiojodtherapie aus, welches die nachfolgenden Kontrolluntersuchungen erschwert.

Im Zweifelsfall ist die Hemithyreoidektomie aber vertretbar und kann die Patienten mit in die Entscheidungsfindung für oder gegen eine 2-zeitige vollständige Thyreoidektomie einbinden. Letzteres wird in den ATA-Leitlinien besonders hervorgehoben. Auch nach den ATA-Leitlinie 2015 wird die vollständige Thyreoidektomie in folgenden Situationen empfohlen:

- bei Vorliegen von 1 oder mehreren Risikofaktoren (Alter > 55 Jahre, männlich, multiple > 3 PTMC, Verwandter 1. Grades mit PTC/FTC, BRAF+, R1),

- bei größeren papillären und follikulären Tumoren,

- bei makroskopischer Infiltration der Schilddrüsenkapsel und

- bei allen aggressiven Varianten des papillären und follikulären (breit invasives FTC) Schilddrüsenkarzinoms.

Eine zusätzliche systematische zentrale Lymphknotendissektion ist bei klinisch nachweisbaren Lymphknotenmetastasen (Zytologie plus Thyreoglobulin im Aspirat) und bei klinisch unauffälligen Befunden für alle T3-Tumoren und alle aggressiven PTC-Varianten (BRAF+, tall cell), allen medullären Schilddrüsenkarzinomen (MTC) sowie allen wenig differenzierten Schilddrüsenkarzinomen (PDTC etc.) indiziert.

Einen nachweislichen Einfluss auf das Gesamtüberleben hat die chirurgische Intervention jedoch nur bei Hochrisikopatienten mit PTC und FTC sowie bei Patienten mit MTC und PDTC. Das Risiko wird dabei bestimmt durch das Patientenalter, die Histologie des Tumors (aggressive Varianten des PDTC, grobinvasives FTC), das Auftreten großflächigen extrathyreoidalen Tumorwachstums, den Nachweis von Lymphknoten- und Fernmetastasen sowie die Vollständigkeit der Tumorentfernung.

So hat in Deutschland die bevorzugte Thyreoidektomie (TX) bei allen Patienten mit PTC ab einer Tumorgröße $>1 \mathrm{~cm}$ und bei fast allen Patienten mit einem follikulären Karzinom (Ausnahme minimalinvasives FTC $<2 \mathrm{~cm}$ ) sowie die relativ weite Indikation zur postoperativen Radiojodtherapie (bei allen Patienten mit TX wegen eines PTC/FTC über dem 
45. Lebensjahr) dazu geführt, dass die Zahl übersehener Fernmetastasen als sehr gering angenommen werden kann. Dies trifft nicht für alle Fälle eingeschränkter Operationsradikalität zu, wie es aufgrund der SEER-Überlebensdaten in den ATA-Leitlinien 2015 empfohlen wird.

\section{KERNAUSSSAGEN}

- In Deutschland und in ehemaligen Jodmangelgebieten Europas sind Schilddrüsenknoten und Knotenstruma immer noch sehr viel häufiger als in den USA.

- Die Leitlinien der Amerikanischen Schilddrüsengesellschaft (ATA) geben Empfehlungen für die klinische und sonografische Abklärung, einschließlich der Indikation zur einer Feinnadelaspirationszytologie, von solitären Knoten und Knoten in einer Struma.

- Einzelne Kriterien, die auf Malignität hinweisen können, gelten für solitäre Knoten, sind aber für Knoten einer Knotenstruma nur bedingt verwertbar: So ergibt sich etwa bei einem schlecht abgrenzbaren Knoten mit unscharfem Rand in einer Struma mit ineinander verwachsenen Knoten häufig kein Hinweis auf Malignität. Auch Echoarmut ist für benigne Knoten in ehemaligen Jodmangelgebieten relativ charakteristisch und erst eine ausgeprägte Echoarmut eines Knotens kann auf Malignität hindeuten.

- Ein weiterer Unterschied betrifft die Durchführung einer Szintigrafie: Die ATA empfiehlt eine I-123-Szintigrafie zur Abklärung eines Knotens nur, wenn das TSH supprimiert ist. Einige Studien in Jodmangelgebieten haben jedoch gezeigt, dass auch bei einem niedrig normalen TSH-Wert eine Autonomie vorliegen kann. In Deutschland wird deshalb zur präoperativen Abklärung eines Knotens eine Szintigrafie mit Technetium-99m durchgeführt, das gegenüber Jodid-123 den Vorteil einer geringeren Halbwertszeit und einer geringeren Strahlendosis hat.

\section{Interessenkonflikt}

Die Autorinnen/Autoren geben an, dass kein Interessenkonflikt besteht.

\section{Autorinnen/Autoren}

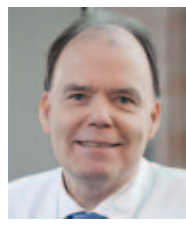

\section{Prof. Dr med. Prof. h. c. Karl-Michael} Derwahl

Ärztlicher Direktor und Chefarzt am St.-Hedwig-Krankenhaus Berlin-Mitte 2001-2018. Seit 2019 Praxis für Endokrinologie und Diabetologie und Leiter des Instituts für klinische Forschung und Entwicklung Berlin.

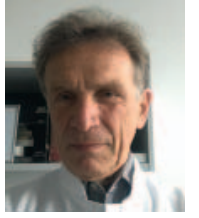

Prof. Dr. med. Dr. h. c. Peter E. Goretzki

2001-2017 Chefarzt der Allgemein-, Gefäß und Thoraxchirurgie Lukas-Krankenhaus Neuss; seit 2018 Leiter Endokrine Chirurgie, CharitéUniversitätsmedizin Berlin; CCM/CVK.

\section{Korrespondenzadresse}

\section{Prof Dr. med. Karl-Michael Derwahl}

Endokrinologie und Diabetologie und Institut für klinische Forschung und Entwicklung Berlin

Gethsemanestr. 6

10437 Berlin-Prenzlauer Berg

derwahl@ikfe-berlin.de

\section{Literatur}

[1] Haugen BR, Alexander EK, Bible KC et al. American Thyroid Association management guidelines for adult patients with thyroid nodules and differentiated thyroid cancer: the American Thyroid Association guidelines task force on thyroid nodules and differentiated thyroid cancer. Thyroid 2016; 26 : $1-133$

[2] Bundesministerium für Ernährung und Landwirtschaft. Jodversorgung in Deutschland: Ergebnisse des Jodmonitorings. Verfügbar unter (Aufgerufen am 6.4.2020): https://www. bmel.de/DE/Ernaehrung/GesundeErnaehrung/_Texte/ DEGS_JodStudie.html

[3] Reiners C, Wegscheider K, Schicha $\mathrm{H}$ et al. Prevalence of thyroid disorders in the working population of Germany: ultrasonography screening in 96,278 unselected employees. Thyroid 2004; 14 (11): 926-932

[4] Luster M, Aktolun C, Amendoeira I et al. European Perspective on 2015 American Thyroid Association Management Guidelines for Adult Patients with Thyroid Nodules and Differentiated Thyroid Cancer: Proceedings of an Interactive International Symposium. Thyroid 2019; 29 (1): 7-26

[5] Ito Y, Miyauchi A, Oda H. Low-risk papillary microcarcinoma of the thyroid: A review of active surveillance trials. Eur J Surg Oncol 2018; 44 (3): 307-315

[6] Nutz V, Larena-Avellaneda A, Wunsch E et al. Frequency of malignancy and indications for surgery in nodular goiter in an endemic area. Dtsch Med Wochenschr 1984; 109 (35): 13191321

[7] Holzer S, Reiners C, Mann K et al. Patterns of care for patients with primary differentiated carcinoma of the thyroid gland treated in Germany during 1996. U.S. and German Thyroid Cancer Group. Cancer 2000; 89 (1): 192-201

[8] Feldt-Rasmussen U. lodine and cancer. Thyroid 2001; 11 (5): 483-486

[9] Chami R, Moreno-Reyes R, Corvilain B. TSH measurement is not an appropriate screening test for autonomous functioning thyroid nodules: a retrospective study of 368 patients. Eur J Endocrinol 2014; 170 (4): 593-599

[10] Graf D, Helmich-Kapp B, Graf S et al. Functional activity of autonomous adenoma in Germany. Dtsch Med Wochenschr 2012; 137 (41): 2089-2092

[11] Karges W, Dralle H, Raue F et al. Calcitonin measurement to detect medullary thyroid carcinoma in nodular goiter: German evidence-based consensus recommendation. Exp Clin Endocrinol Diabetes 2004; 112 (1): 52-58 
[12] Frank-Raue K, Schott M, Raue F et al. Recommendation for Calcitonin Screening in Nodular Goiter. Dtsch Med Wochenschr 2018; 143 (15): 1065-1069

[13] Shin JH, Baek JH, Chung J et al. Ultrasonography Diagnosis and Imaging-Based Management of Thyroid Nodules: Revised Korean Society of Thyroid Radiology Consensus Statement and Recommendations. Korean J Radiol 2016; 17 (3): 370-395

[14] Russ G, Bonnema S], Erdogan MF et al. European Thyroid Association Guidelines for Ultrasound Malignancy Risk Stratification of Thyroid Nodules in Adults: The EU-TIRADS. Eur Thyroid J 2017; 6 (5): 225-237

[15] Grünwald F, Derwahl KM. Diagnostik und Therapie von Schilddrüsenerkrankungen. 3 Aufl Berlin: Lehmanns Media; 2019

[16] Rago T, Fiore E, Scutari M et al. Male sex, single nodularity, and young age are associated with the risk of finding a papillary thyroid cancer on fine-needle aspiration cytology in a large series of patients with nodular thyroid disease. Eur J Endocrinol 2010; 162 (4): 763-770

[17] McLeod DS, Watters KF, Carpenter AD et al. Thyrotropin and thyroid cancer diagnosis: a systematic review and doseresponse meta-analysis. J Clin Endocrinol Metab 2012; 97 (8): 2682-2692

[18] Leenhardt L, Erdogan MF, Hegedus L et al. 2013 European thyroid association guidelines for cervical ultrasound scan and ultrasound-guided techniques in the postoperative management of patients with thyroid cancer. Eur Thyroid J 2013; 2 (3): 147-159

[19] Crippa S, Mazzucchelli L, Cibas ES et al. The Bethesda System for reporting thyroid fine-needle aspiration specimens. Am J Clin Pathol 2010; 134 (2): 343-344; author reply 345
[20] Cheung L, Messina M, Gill A et al. Detection of the PAX8-PPAR gamma fusion oncogene in both follicular thyroid carcinomas and adenomas. J Clin Endocrinol Metab 2003; 88 (1): 354-357

[21] Nikiforov YE. Ramifications of New Terminology for Encapsulated Follicular Variant of Papillary Thyroid Carcinoma-Reply. JAMA Oncol 2016; 2 (8): 1098-1099

[22] Francis DO, Randolph G, Davies L. Nationwide variation in rates of thyroidectomy among US medicare beneficiaries. JAMA Otolaryngol Head Neck 2017; 143: 1122-1125

[23] Bartsch DK, Luster M, Buhr H] et al. Indication for the surgical management of benign goiter in adults. Dtsch Arztebl Int 2018; 115: 1-7

[24] Dralle H, Musholt T, Schabram J et al. German Association of Endocrine Surgeons practice guidelines for the management of malignant thyroid tumors. Langenbecks Arch Surg 2013; 398: $347-374$

[25] Ho AS, Luu M, Zalt C et al. Mortality risk of nonoperative papillary thyroid carcinoma: a corollary active surveillance. Thyroid 2019; 29: 1409-1417

[26] Albano D, Bertagna F, Bonacina M et al. Possible delayed diagnosis and treatment of metastatic differentiated thyroid cancer by adopting the 2015 ATA guidelines. Eur J Endocrinol 2018; 179: 143-151

\section{Bibliografie}

DOI https://doi.org/10.1055/a-1018-2767

Online-Publikation: 7.7.2020

Dtsch Med Wochenschr 2020; 145: 1227-1235

(c) Georg Thieme Verlag KG, Stuttgart · New York ISSN 0012-0472 